\title{
Research Paper \\ Bioinformatics Prediction of miRNAs Targeting E6 and E7 Genes in Human Pap- illomavirus Types 16 and 18 in Cervical Cancer
}

\author{
Tahere Azimi $^{1} \odot$, Malihe Bagheri ${ }^{1} \odot$, Mahdi Pariyan², Behzad Khansarinejad ${ }^{3} \odot$, Ashraf Zamani ${ }^{4} \odot{ }^{*}$ Mahdieh Mon- $^{2}$ \\ danizadeh $^{1,5}$
}

1. Department of Biotechnology and Molecular Medicine, School of Medicine, Arak University of Medical Sciences, Arak, Iran.

2. Department of Research and Development, Production and Research Complex, Pasteur Institute of Iran, Tehran, Iran.

3. Department of Microbiology and Immunology, School of Medicine, Arak University of Medical Sciences, Arak, Iran.

4. Department of Obstetrics and Gynecology, School of Medicine, Arak University of Medical Sciences, Arak, Iran.

5. Molecular and Medical Research Center, Arak University of Medical Sciences, Arak, Iran.

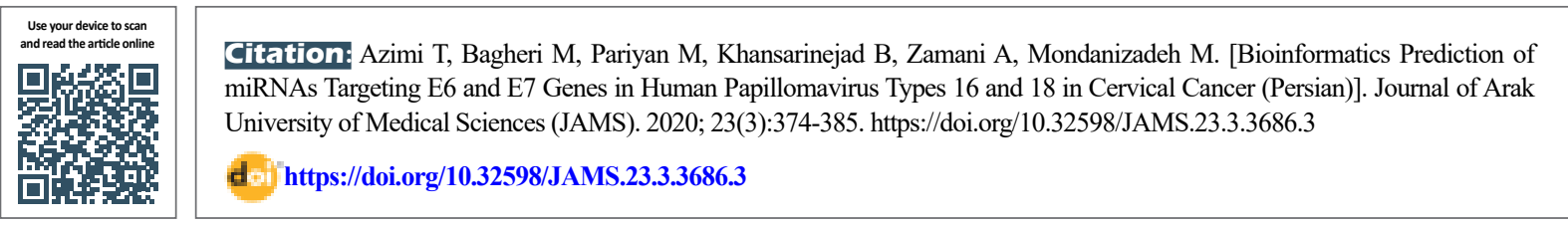

$(\omega)$

Article Info:

Received: 22 Sep 2019

Accepted: 22 Dec 2019

Available Online: 01 Aug 2020

Key words:

Bioinformatics data-

bases, miRNA, Cervi-

cal cancer, Human

papillomavirus

\section{A B STRACT}

Background and Aim Cervical Cancer (CC) is the third most common malignancy in the women, the main cause of which is human papillomavirus (HPV). Both E6 and E7 oncogenes of the virus play an important role in its tumorigenesis. Today, methods available for screening CC are not capable of detecting the disease at an early stage. Therefore, it is important to identify new biomarkers for early detection of this cancer. For this purpose, in the present study, miRNAs targeting the two oncogenes E6 and E7 of human papillomavirus (types 16 and 18) were studied in CC by bioinformatics.

Methods \& Materials First, using the NCBI database, the E6 and E7 gene sequences were obtained for both human papillomavirus types 16 and 18. Then, using the miRBase and RNA22 bioinformatics databases, the most appropriate targeting miRNAs for these genes were selected.

Ethical Considerations This study was approved by Ethics Committee of Arak University of Medical Sciences. Results Based on the $P$ obtained from bioinformatics databases, miRNA including miR-92a-5p $(P=7.51 e-2)$, miR-195-3p ( $P=2.24 e-1)$, miR-34a-5p ( $P=2.73 e-1)$ and miR-155-5p ( $P=4.95 e-2)$ were introduced for the two genes $\mathrm{E} 6$ and $\mathrm{E} 7$.

Conclusion Results from bioinformatics studies revealed that of the four miRNAs identified, miR-155-5p and miR-92a-5p are probably the targeting miRNAs specific for the E6 and E7 genes, respectively. Therefore, it seems that these miRNAs can be a suitable candidate for in vitro studies in CC patients.

\section{Extended Abstract}

\section{Introduction}

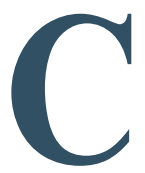

ervical Cancer (CC) is the third most common malignancy in the women, the main cause of which is Human Papillomavirus (HPV) $[3,6]$. The E6 and E7 genes in the
HPV genome are known as viral oncogenes and play an important role in its tumorigenesis [9]. At present, methods available for screening $\mathrm{CC}$ are not capable of detecting the disease at an early stage [13]. Therefore, it is important to identify new and non-invasive biomarkers with high sensitivity and specificity for early detection of this cancer. Moreover, miRNAs are found in different tissues with variable expression, and their expression profiles change in the

\section{* Corresponding Author:}

Mahdieh Mondanizadeh, PhD.

Address: Molecular and Medical Research Center, Arak University of Medical Sciences, Arak, Iran.

Tel: +98 (86) 34172526

E-mail: m mondanizadeh@yahoo.com 
disease state [30]. These molecules can be used as novel biomarkers in diagnosis and treatment of various cancers [31]. Therefore, in the present study, miRNAs targeting the two oncogenes E6 and E7 of human papillomavirus (types 16 and 18) were studied in CC by bioinformatics tools.

\section{Materials and Methods}

First, the E6 and E7 genes sequences were obtained for both human papillomavirus types 16 and 18 using the NCBI database (http://www.ncbi.nlm.nih.gov/gene). Then, the most appropriate miRNAs targeting E6 and E7 genes were selected using various bioinformatics databases including Miranda, Vir-Mir, Targetscan, miRNA path, miRBase and RNA22. The sequences of predicted miRNAs were retrieved from miRBase database (www.mirbase. org). Moreover, the complementary relationship between miRNA and target gene based on P-value was determined by the RNA22 bioinformatics database (http://cm.jefferson. edu/rna22/Interactive/). The $\mathrm{P}$ value represents the random possibility of binding the target miRNA. That is, a lower $\mathrm{P}$ represents a greater chance of binding to the target gene.

\section{Results}

The bioinformatics analyzes indicated that Miranda, Vir-Mir, Targetscan and miRNA path databases were not able to predict miRNAs targeting E6 and E7 viral genes. The obtained results of miRBase and RNA22 databases demonstrated that four miRNAs including miR-92a$5 \mathrm{p} \quad(\mathrm{P}=7.51 \mathrm{e}-2), \quad \mathrm{miR}-195-3 \mathrm{p} \quad(\mathrm{P}=2.24 \mathrm{e}-1), \quad \operatorname{miR}-34 \mathrm{a}-5 \mathrm{p}$ $(\mathrm{P}=2.73 \mathrm{e}-1)$ and miR-155-5p $(\mathrm{P}=4.95 \mathrm{e}-2)$ are the miRNAs that target E6 and E7 genes (Table 1). Among these miRNAs, miR-155-5p $(\mathrm{P}=4.95 \mathrm{e}-2)$ and miR-92a-5p $(\mathrm{P}=7.51 \mathrm{e}-2)$ are likely the specific target miRNAs for the E6 and E7 genes, respectively.

\section{Discussion}

Numerous studies have shown that miRNAs are closely linked to various diseases, including cancer, because these molecules are involved in all biological processes, including cell growth and differentiation, cell cycle regulation, stress response, and apoptosis [16]. The structure and function of miRNAs suggest that the expression of many miRNAs in cancerous tissues changes abnormally compared to normal tissue [30]. Therefore, changing the expression profile of miRNAs can be used as biomarkers to diagnose a wide range of diseases [31]. At present, various methods such as microarray analysis, In Situ Hybridization (ISH), northern blot, and Real Time PCR are used to measure miRNAs. This method is time consuming and expensive, while bioinformatics tools are effective and low-cost methods that can be used to predict the interaction between miRNAs and target genes [32, 33].

In this study, considering the oncogenic role of E6 and E7 genes of human papillomavirus in $\mathrm{CC}$, different bioinformatics databases were used to identify and predict the miRNAs targeting these genes. Bioinformatics results indicated that four miRNAs including miR-92a-5p, miR-195-3p, miR$34 a-5 p$ and miR-155-5p are the miRNAs that target E6 and E7 genes. Among these miRNAs, miR-155-5p and miR92a-5p are likely the specific target miRNAs for the E6 and E7 genes, respectively. Other studies have shown that eight miRNAs, including miR-16, miR-25, miR-92a, miR-378, miR-22, miR-27a, miR-29a, and miR-100, have the highest accuracy for $\mathrm{CC}$ detection, and they can be used to differentiate between CC patients and healthy individuals [39].

\section{Conclusion}

Our results suggest that miR-155-5p and miR-92a-5p can be a suitable candidate for in vitro studies in $\mathrm{CC}$ patients.

Table 1. All selected miRNAs for evaluation of expression level in patient with cervical cancer

\begin{tabular}{ccc}
\hline Gene & miRNA & ID \\
\hline E6 (HPV16) & has-miR-155-5p & MIMAT0000646 \\
\hline E6 (HPV18) & has-miR-195-3p & MIMAT0004615 \\
\hline E7 (HPV16) & has-miR-34a-5p & MIMAT0000255 \\
\hline E7 (HPV18) & has-miR-92a-5p & MIMAT0004507 \\
\hline
\end{tabular}


These miRNAs can be used as diagnostic biomarkers if they have a change in expression in $\mathrm{CC}$ patients compared to healthy individuals.

\section{Ethical Considerations}

Compliance with ethical guidelines

This study approved in Ethical Committee of Arak University of Medical Science (Code: IR.ARAKMU. REC.1396.296).

Funding

TThe present paper was extracted from the MSc thesis of the first author, Department of Biotechnology and Molecular Medicine, School of Medicine, Arak University of Medical Sciences.

\section{Authors' contributions}

All authors contributed in preparing this article.

\section{Conflicts of interest}

The authors declared no conflict of interest.

\section{Acknowledgements}

The authors would like to thank Research Assistance of Arak University of Medical Sciences due to its financial grant and support of this study. 


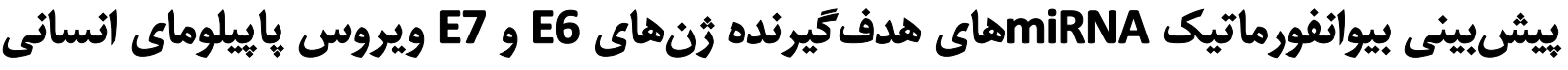

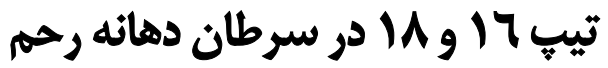

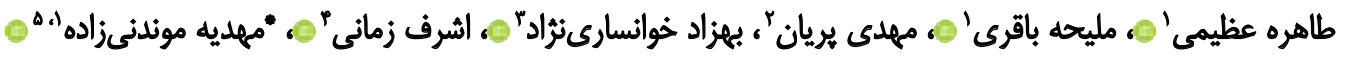 \\ ا. كروه زيست فناورى و يزشكى مولكولى، دانشكده يزشكى، دانشعاه علوميزشكى اراكى اراكى ايران.

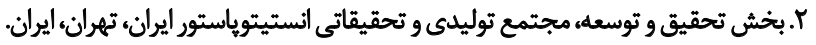

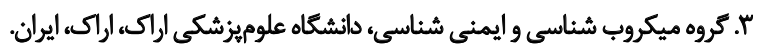

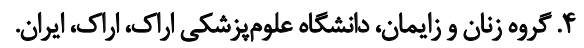

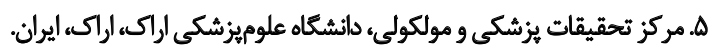

إنمينه و هدف سرطان دهانه رحم (CC)

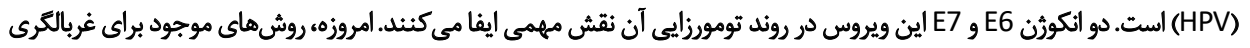

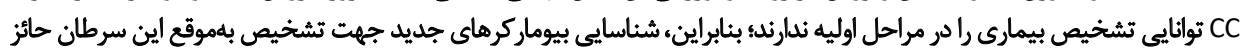

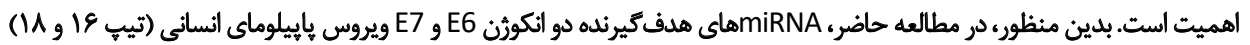

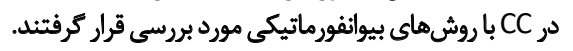

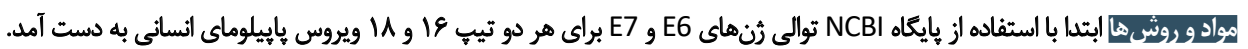

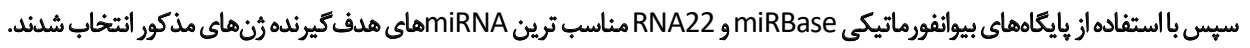

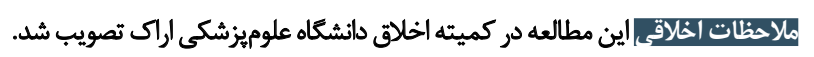

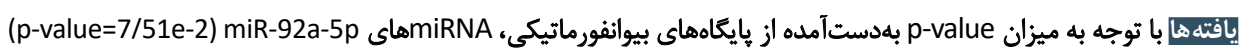
miR-155-5p (p-value=4.95e-2), miR-195-3p (p-value=2.24e-1) ،miR-34a-5p (p-value=2.73e-1) E6 , E6 Eعرفى شدئد.

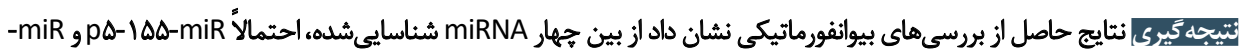

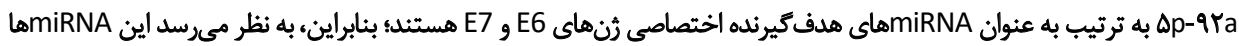

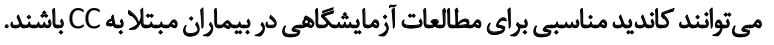

اطلاعات مقاله:

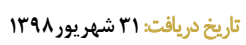

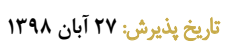

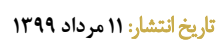

كليدوأرها:

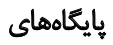

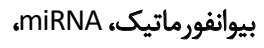

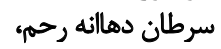
ويروس يا إييلوماي دانه انسانى

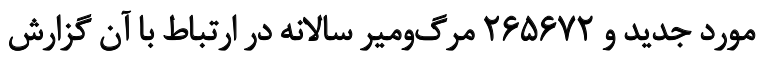

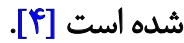

عوامل مؤثر در ابتلا به اين سرطان ويروس يإييلوماى انسانى

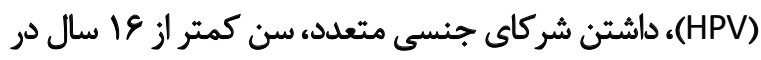

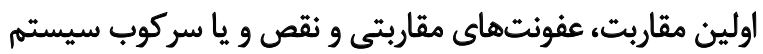

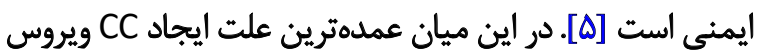

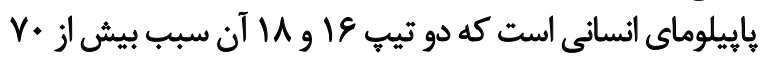

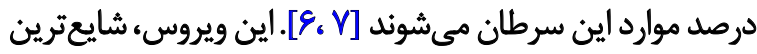

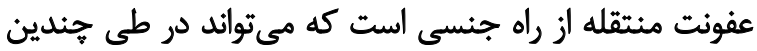

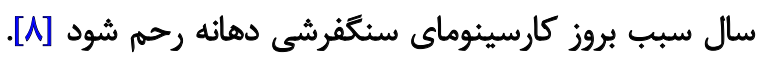

doles

سرطان يكى از مهمترين مشكلات بهداشت عمومى در سراسر

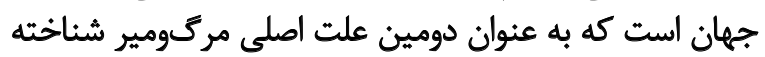

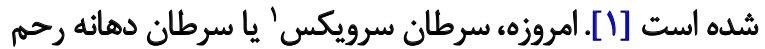

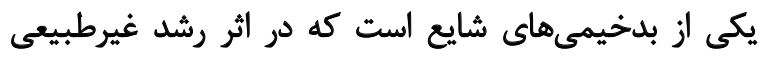

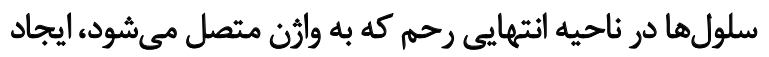

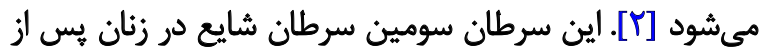

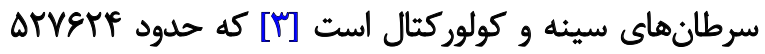

1. Cervical Cancer (CC)

* نويسئده مسئول:

مهيديه موندنى نواده

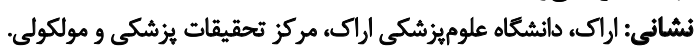
تلفن: يست الكترونيكي: m_mondanizadeh@yahoo.com 


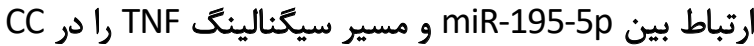

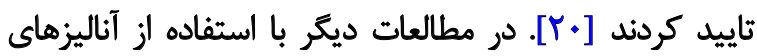

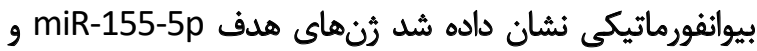

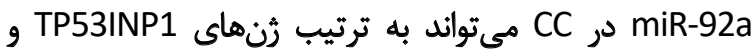

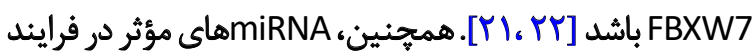

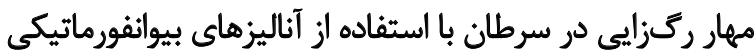

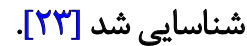
در اين مطالعه نيز با استفاده از ابزارهاى بيوانفورماتيكى

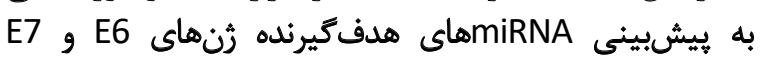

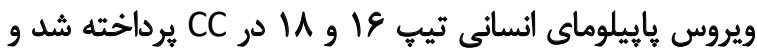

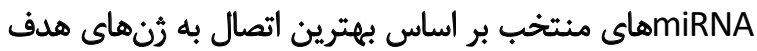

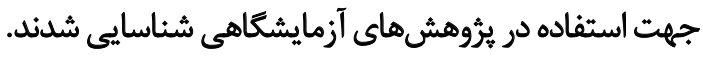

\section{مواد و روشها}

يرؤشش حاضر يك بررسى تئورى بيوانفورماتيك است.

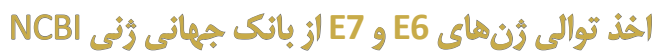

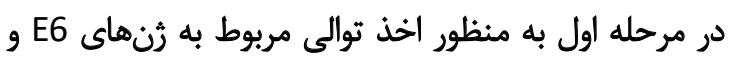

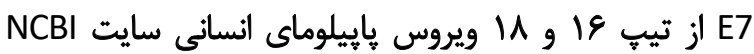
مورد استفاده قرار (http://www.ncbi.nlm.nih.gov/gene)

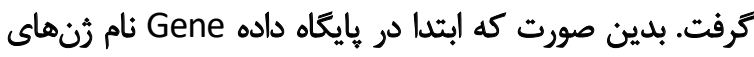

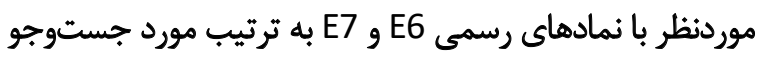

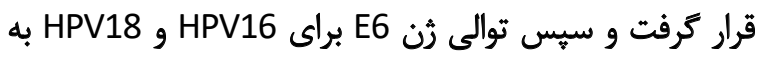

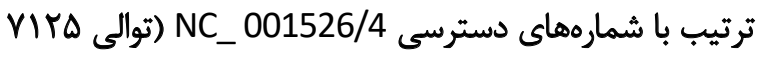

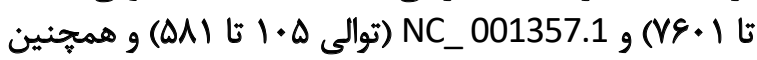
توالى ثن E7 براى HPVIS

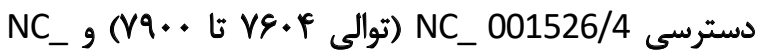

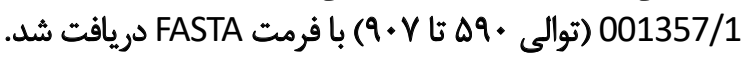

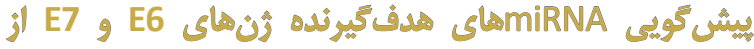

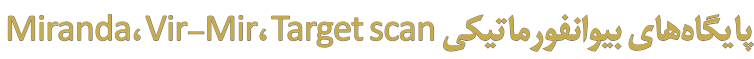 miRNA path g}

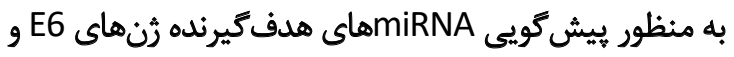

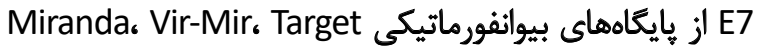

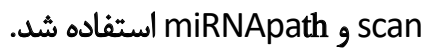

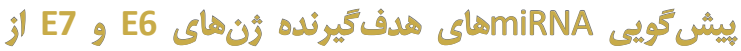

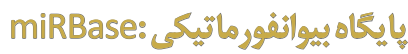

إِايكاهاطلاعاتى (www.mirbase.org) miRBase يك منبع

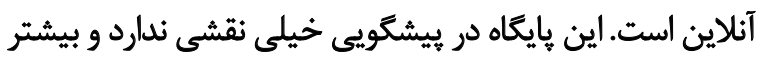

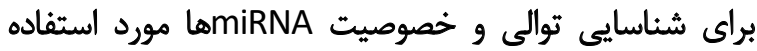

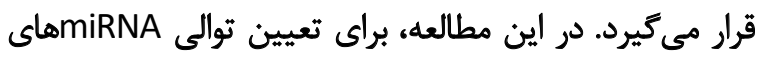

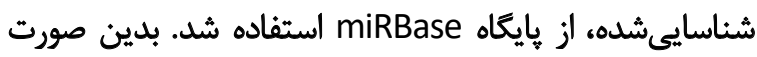

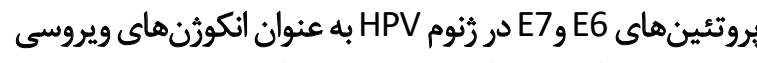

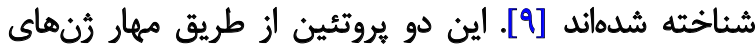

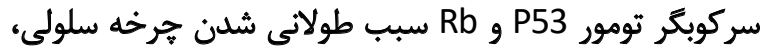

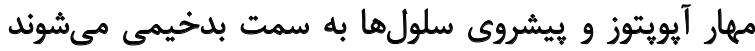

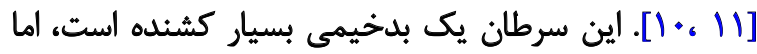

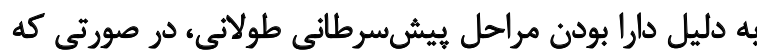

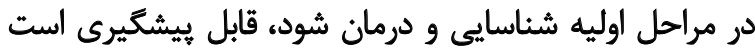

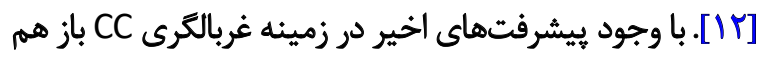

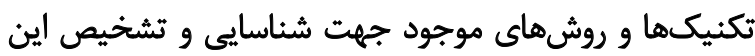

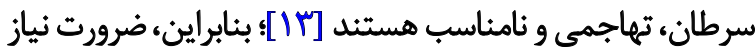

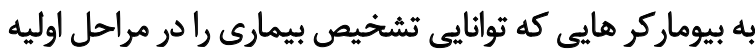

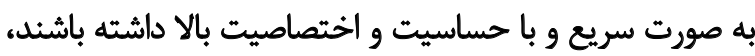
احساس مىشود.

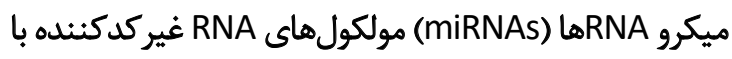

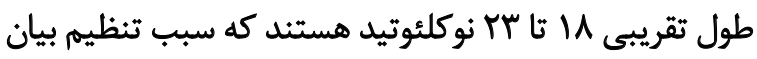

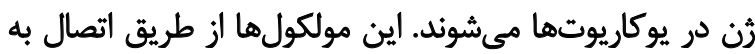

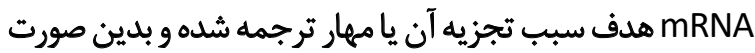

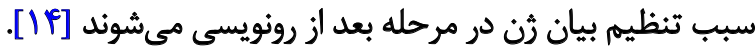

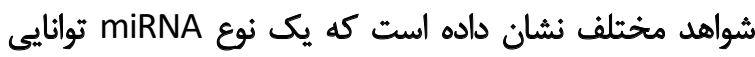

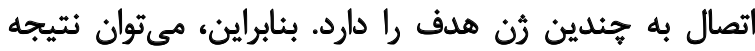

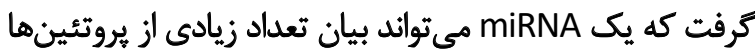

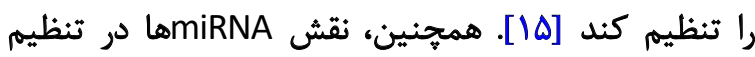

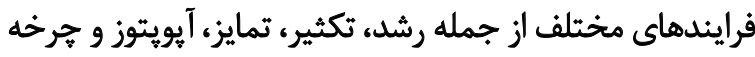

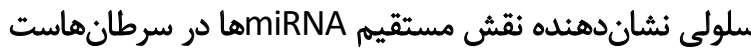

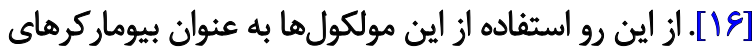

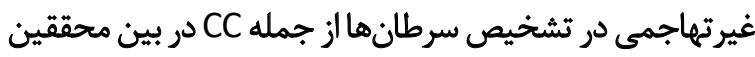
بسيار مورد توجه قرار ترفتئه است.

از طرفى استفاده از يايكاههاي بيوانفورماتيكى متعدد براي

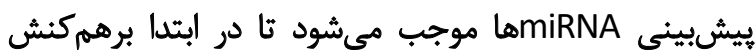

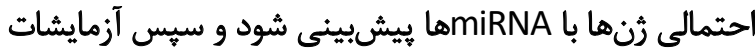

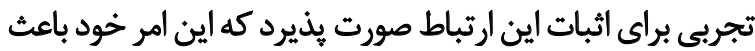

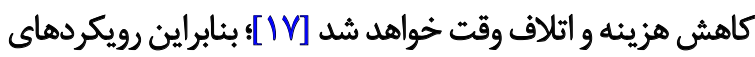

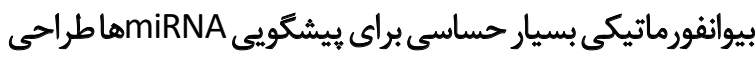

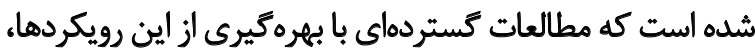

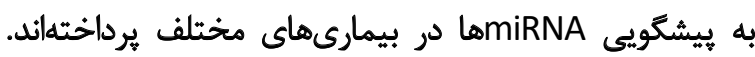

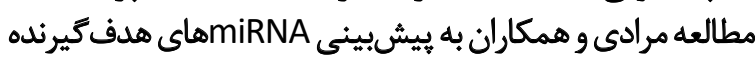

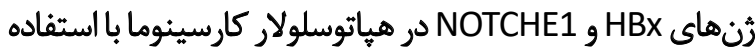

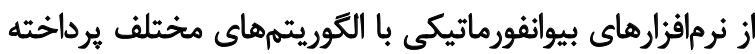

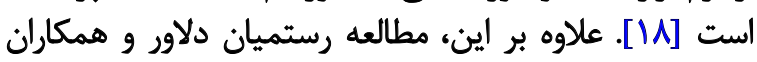

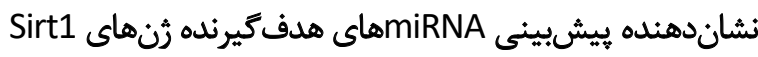

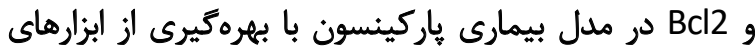

بيوانفورماتيكى بود [19] داري

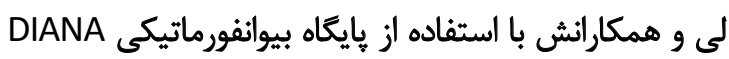


كه در قسمث By miRNA identifier or keyword نام هر miRNA وارد شد و بعد از submit و انتخاب miRNA موردنظر

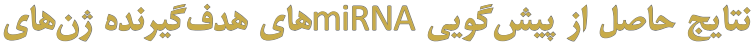

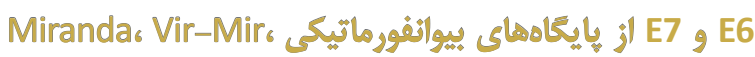
miRNA path, Target scan

در اين مطالعه از بايكاههاى بيوانفورماتيكى متعددى از miRNA path و Mمله Miranda، Vir-Mir ،Target scan

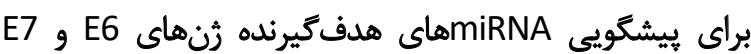

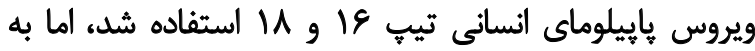

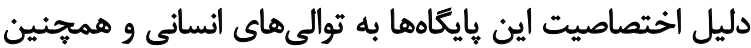

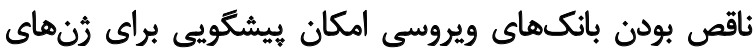

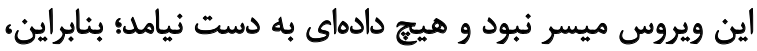
با استفاده از نتايج مطالعات يُ يشين،

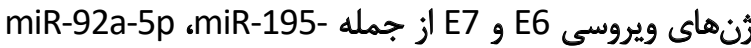

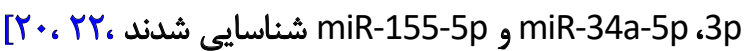

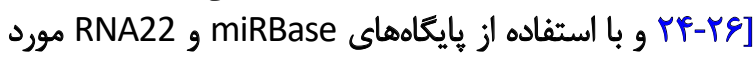

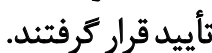

$$
\text { توالى مربوط به آن دريافت شد. }
$$

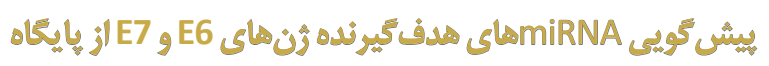

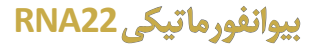

http://cm.jefferson. اين بايكاه اطلاعاتى از طريق سايت

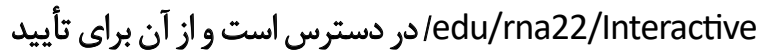

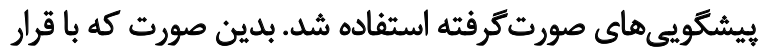

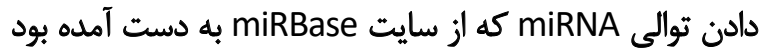

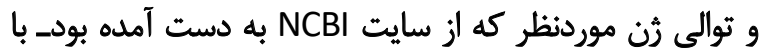

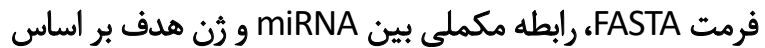

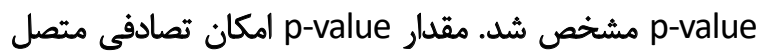

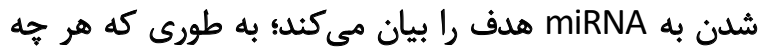

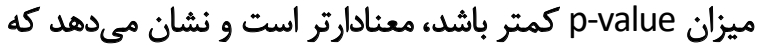

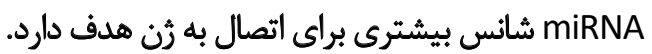

جدول ا. نتايج يُيكويى miR-92a-5p هدف كيرنده ثن E7 در بايكاه RNA22

\begin{tabular}{ccc}
\hline P & Type HPV & miRNA \\
- & HPV16 E6 & miR-92a-5p \\
\hline $7.51 e-2$ & HPV16 E7 & miR-92a-5p \\
- & HPV18 E6 & miR-92a-5p \\
- & HPV18 E7 & miR-92a-5p \\
\hline
\end{tabular}

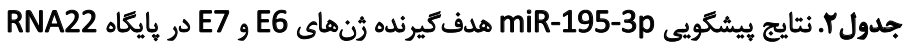

\begin{tabular}{ccc}
\hline P & Type HPV & miRNA \\
\hline- & HPV16 E6 & miR-195-3p \\
\hline $2.24 \mathrm{e}-1$ & HPV16 E7 & miR-195-3p \\
$3.99 \mathrm{e}-2$ & HPV18 E6 & miR-195-3p \\
- & HPV18 E7 & miR-195-3p \\
\hline
\end{tabular}


جدول r. نتايج بيش كويى miR-34a-5p هدفئينده رنهاي E6 و E7 در بايكاه RNA22

\begin{tabular}{ccc}
\hline P & Type HPV & miRNA \\
\hline- & HPV16 E6 & miR-34a-5p \\
- & HPV16 E7 & miR-34a-5p \\
$2.73 e-1$ & HPV18 E6 & miR-34a-5p \\
$5.33 e-2$ & HPV18 E7 & miR-34a-5p \\
\hline
\end{tabular}

جدول f. نتايج بيشكويي miR-155-5p هدف كيرنده رنز E6 در هايكاه RNA22

\begin{tabular}{ccc}
\hline P & Type HPV & miRNA \\
\hline $4.95 e-2$ & HPV16 E6 & miR-155-5p \\
- & HPV16 E7 & miR-155-5p \\
- & HPV18 E6 & miR-155-5p \\
- & HPV18 E7 & miR-155-5p \\
\hline
\end{tabular}

جدول ه. كليه miRNA هاى انتخابشده جهت بررسى ميزان بيان در افراد مبتلا به سرطان دهانه رحم

شماره شيناسايي

MIMAT0000646

MIMAT0004615

MIMAT0000255

MIMAT0004507

MIMAT0004615

MIMAT0000255

\section{miRNA}

has-miR-155-5p

has-miR-195-3p

has-miR-34a-5p

has-miR-92a-5p

has-miR-195-3p

has-miR-34a-5p
نام ن)

E6 (HPV16)

E6 (HPV18)

E7 (HPV16)

E7 (HPV18)

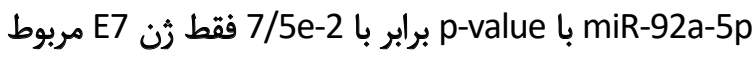

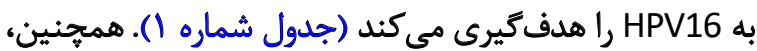

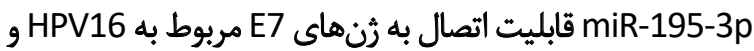

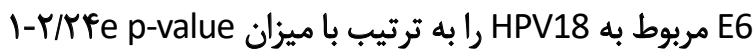

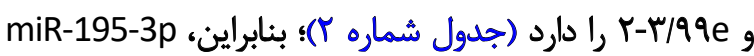

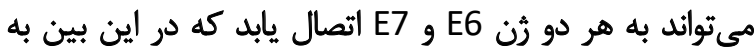

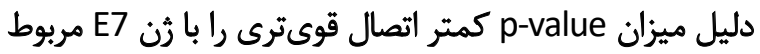

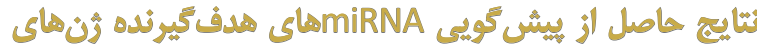

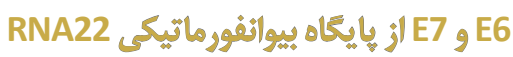

ويشكويى miRNA هدف تيرنده زنهاى E6 و E7 در دو تيب

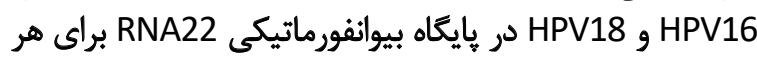
miR-92a-5p ،miR-195-3p، miR-) موردنظي (miR-155-5p , 34a-5p صورت كرفت. اين نتايج نشان داد كه 


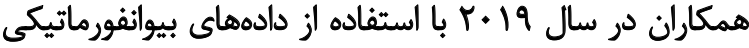

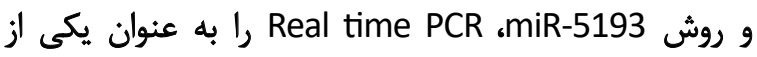

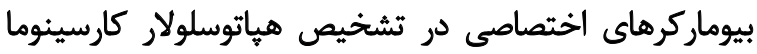

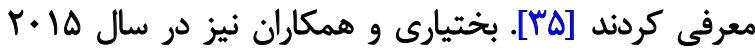

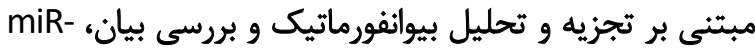

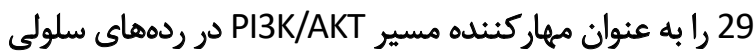

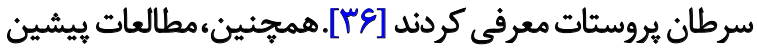

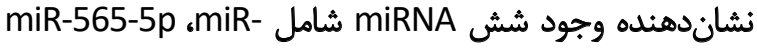
454-5p ،miR-6841-3p،miR-4779-3p ،miR-6779-3p

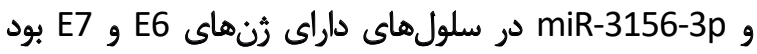

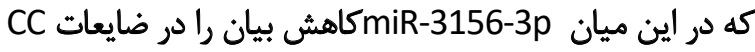

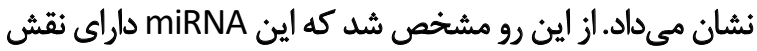

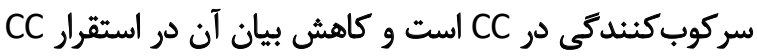

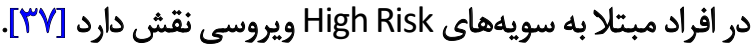

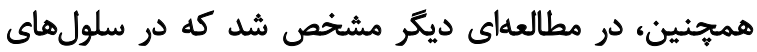

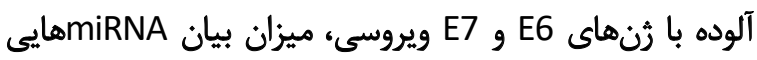

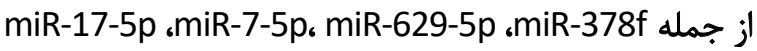
هايى miRNA كاهش يافت miR-186-5p ، ، از جمله miR- و miR-27b-3p ،miR-23b-3p،miR-23a-3p

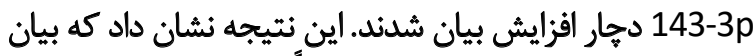

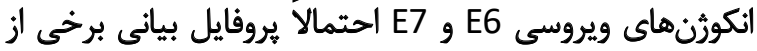
miRNA از طرفى در مطالعه لوئيس و همكارانش نمونههاى داراى

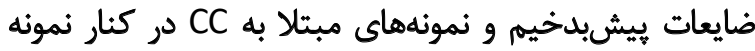

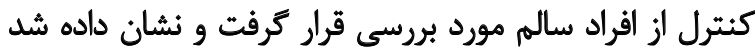

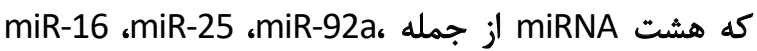
miR-378، miR-22 ،miR-27a ،miR-29a بالاترين دقت براى تشخيص CC هستند و مي توانئد براى تمايز

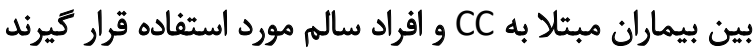

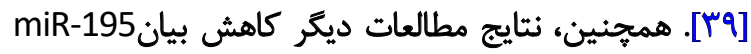

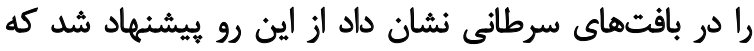

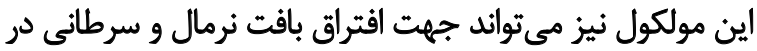

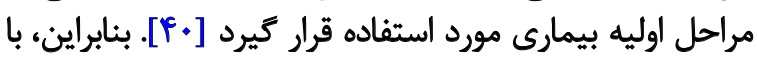

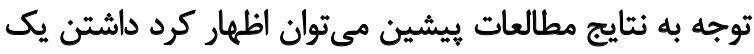

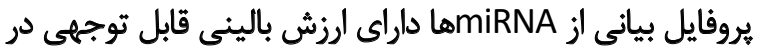
تشخيص CC - (CC است.

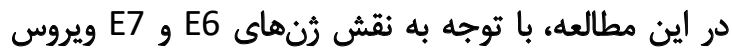

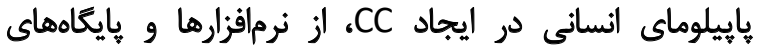

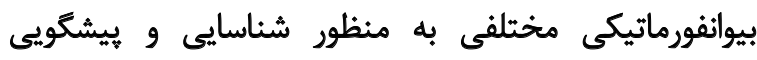

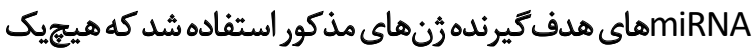

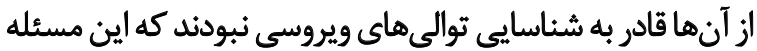

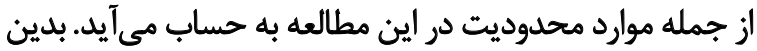

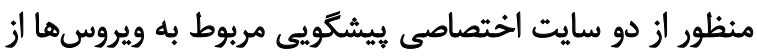

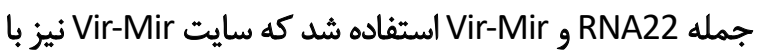

به HPV16 نسبث به E6 مربوط به HPV18 مدارد. هرجه ميزان

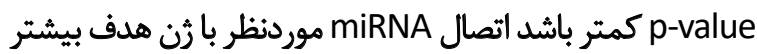

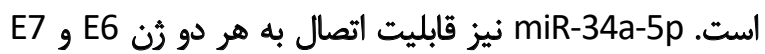

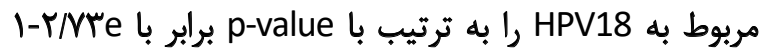

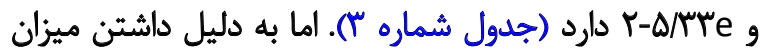

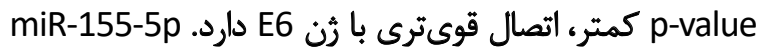

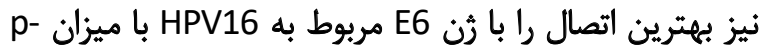

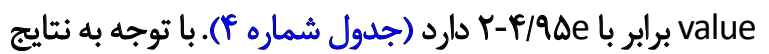

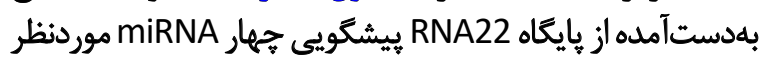
miR-155- giR-92a-5p، miR-195-3p، miR-34a-5p)

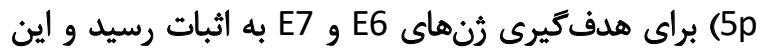

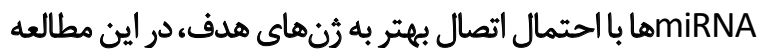
انتخاب شدند (جدول شماره ه).

ث્! سرطان دهانه رحم يكى از شايعترين بدخيمىها در سراسر

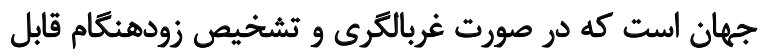

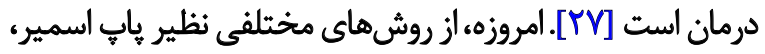

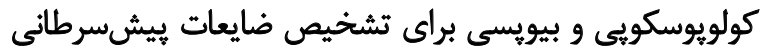

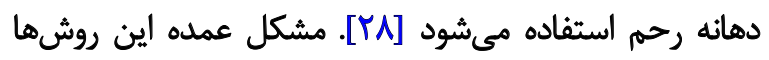

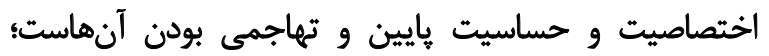

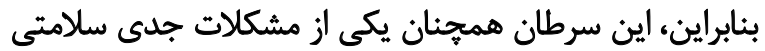

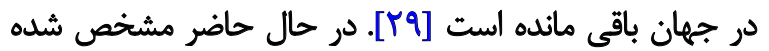

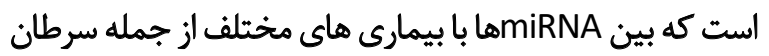

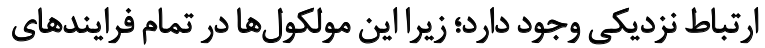

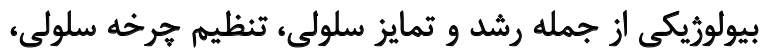

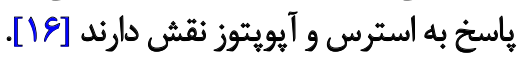

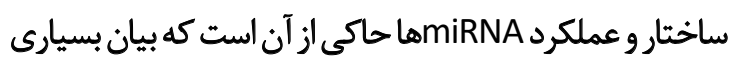

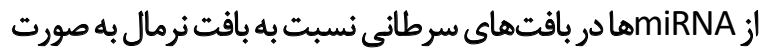

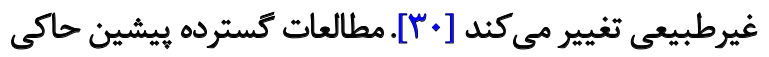

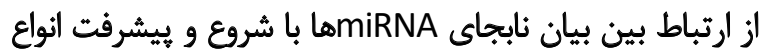

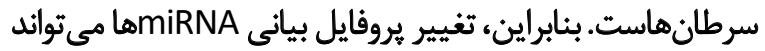

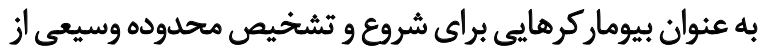

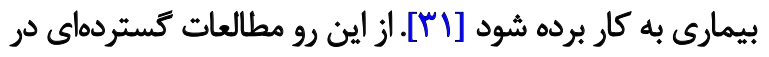

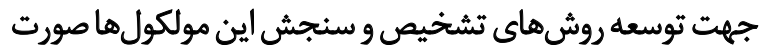

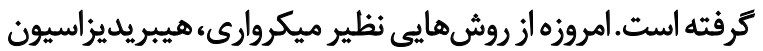

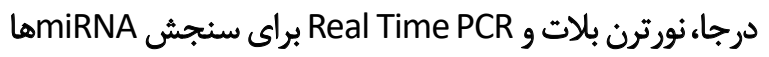

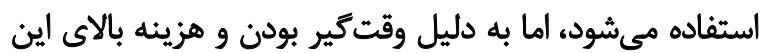

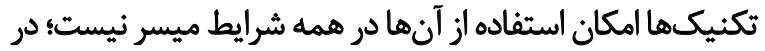

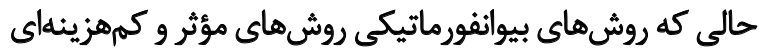

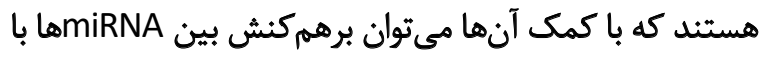

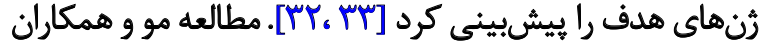

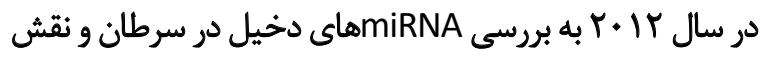

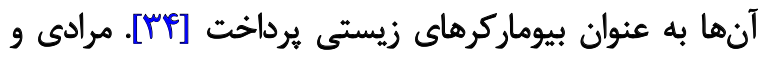




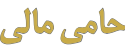

اين مقاله مستخرج از إيان نامه مقطع كارشناسى ارشد نويسنده

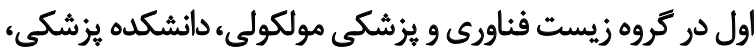

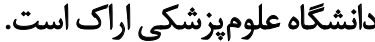

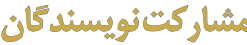

تمامى نويسندكان در نكارش اين مقاله مشاركت داشتهاند.

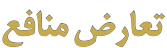

نويسندكان مقاله هيجگ كونه تعارضى در منافع اعلام نكردند.

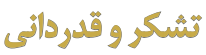

تمامى نويسندكان مراتب قدردانى خود رالز همكار ان محترم در

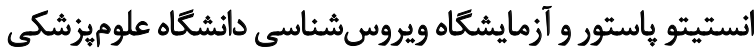

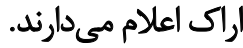

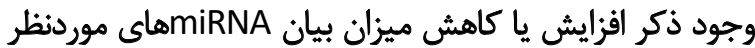

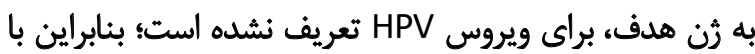

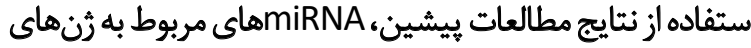
E6 و E7 ويروسى شناسايى شدند.

نتايج اين مطالعات حاكى از تغيير بيان miRNA دايى از جمله

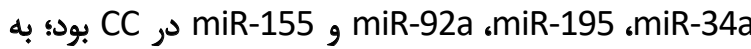
miR-92a كاهش و بيان miR-195وmiR-34a طورى كث بيان

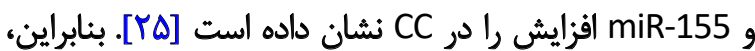

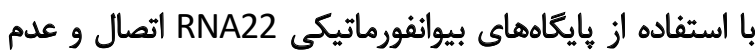

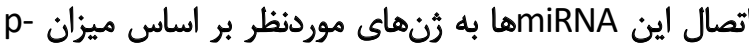
هاى هدفئيرنده

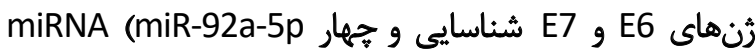
(miR-155-5p و به عنوان بهترين كانديدا براى رُنهاي موردنظر انتخاب شديند. سطح بيان

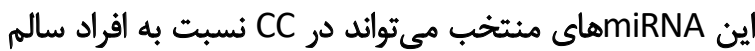

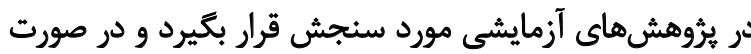

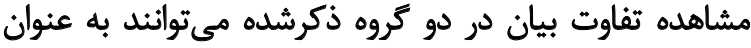

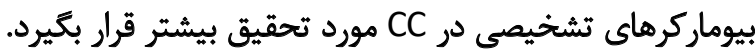

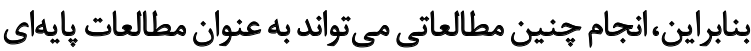

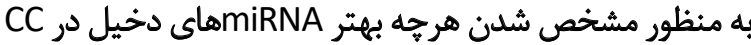

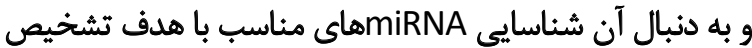
سرطان سرويكس حائز اهميت باشد.

$$
\text { نتيجنيرى }
$$

با توجه به نتايج بهدستآمده از يايكاههاى بيوانفورماتيكى

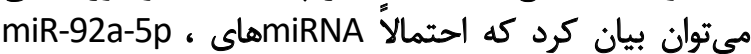
من miR-155-5p , miR-195-3p ،miR-34a-5p مناسب براى دو رُن E6 و E7 ويروس يإييلوماي انسانى هستئد.

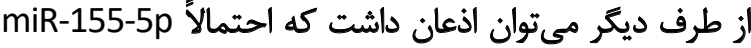

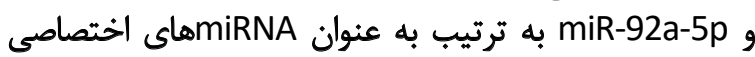

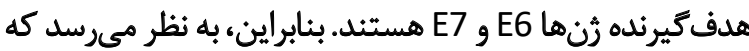

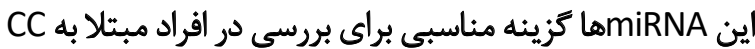

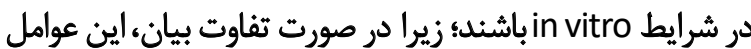

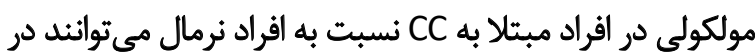
جهت تشخيص در اين سرطان به كار برده شوند.

مالاحظات اخلاقى

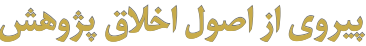

اين مطالعه در كميته اخلاق دانشكاه علوميزشكى اراك با كد

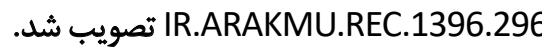




\section{References}

[1] Torre LA, Islami F, Siegel RL, Ward EM, Jemal A. Global cancer in women: burden and trends. Cancer Epidemiol Biomarkers Prev. 2017; 26(4):44457. [DOI:10.1158/1055-9965.EPI-16-0858] [PMID]

[2] Trott P. International classification of diseases for oncology. J Clin Pathol Suppl Coll Pathol. 1977; 30(8):782. [DOI:10.1136/jpp.30.8.782-c] [PMCID]

[3] Ferlay J, Shin HR, Bray F, Forman D, Mathers C, Parkin DM. Estimates of worldwide burden of cancer in 2008: GLOBOCAN 2008. Int J Cancer. 2010; 127(12):2893-917. [DOI:10.1002/ijc.25516] [PMID]

[4] Shrestha AD, Neupane D, Vedsted P, Kallestrup P. Cervical cancer prevalence, incidence and mortality in low and middle income countries: A systematic review. Asian Pac J Cancer Prev. 2018; 19(2):319-24. [DOI:10.22034/APJCP.2018.19.2.319]

[5] Kashyap N, Krishnan N, Kaur S, Ghai S. Risk factors of cervical cancer: A case-control study. Asia Pac J Oncol Nurs. 2019; 6(3):308-14. http:// www.apjon.org/text.asp?2019/6/3/308/255384

[6] Cox JT. Human papillomavirus testing in primary cervical screening and abnormal Papanicolaou management. Obstet Gynecol Surv. 2006; 61(6):S15-S25. [DOI:10.1097/01.ogx.0000221011.01750.25] [PMID]

[7] Clifford G, Smith J, Plummer M, Munoz N, Franceschi S. Human papillomavirus types in invasive cervical cancer worldwide: A meta-analysis. Br J Cancer. 2003; 88(1):63-73. [DOI:10.1038/ sj.bjc.6600688] [PMID] [PMCID]

[8] Khodakarami N, Farzaneh F, Yavari P, Khayamzadeh M, Taheripanah R, Esmaeil Akbari M. [The new guideline for cervical cancer screening in low risk Iranian women (Persian)]. Iran J Obstet Gynecol Infertility. 2014; 17(95):8-17. [DOI:10.22038/IJOGI.2014.2801]

[9] Narisawa-Saito M, Kiyono T. Basic mechanisms of high-risk human papillomavirus-induced carcinogenesis: Roles of E6 and E7 proteins. Cancer Sci. 2007; 98(10):1505-11. [DOI:10.1111/ j.1349-7006.2007.00546.x] [PMID]

[10] Wallace NA, Galloway DA. Novel functions of the human papillomavirus E6 oncoproteins. Annu Rev Virol. 2015; 2:403-23. [DOI:10.1146/ annurev-virology-100114-055021] [PMID]

[11] Mittal S, Banks L. Molecular mechanisms underlying human papillomavirus E6 and E7 oncoprotein-induced cell transformation. Mutat Res Rev Mutat Res. 2017; 772:23-35. [DOI:10.1016/j. mrrev.2016.08.001] [PMID]

[12] Ndikom CM, Ofi BA. Pre-screening counseling in cervical cancer prevention: Implications for nursing. Int Jof Nurs Midwifery. 2011;

3(10):158-64. http://citeseerx.ist.psu.edu/viewdoc/download?doi=10 . 1.1.1027.9690\&rep=rep1\&type=pdf

[13] Reddy KB. MicroRNA (miRNA) in cancer. Cancer Cell Int. 2015; 15(1):38. [DOI:10.1186/s12935-015-0185-1] [PMID] [PMCID]

[14] Cai Y, Yu X, Hu S, Yu J. A brief review on the mechanisms of miRNA regulation. Genomics Proteomics Bioinformatics. 2009; 7(4):147-54. [DOI:10.1016/S1672-0229(08)60044-3]

[15] Ben-Hamo R, Efroni S. MicroRNA regulation of molecular pathways as a generic mechanism and as a core disease phenotype. Oncotarget. 2015; 6(3):1594-04. [DOI:10.18632/oncotarget.2734] [PMID] [PMCID]

[16] Esquela-Kerscher A, Slack FJ. Oncomirs-microRNAs with a role in cancer. Nat Rev cancer. 2006; 6(4):259-69. [DOI:10.1038/nrc1840] [PMID]
[17] Riffo-Campos Á, Riquelme I, Brebi-Mieville P. Tools for sequencebased miRNA target prediction: What to choose? Int J Mol Sci. 2016; 17(12):1987. [DOI:10.3390/ijms17121987] [PMID] [PMCID]

[18] Moradi N, Paryan M, Khansarinejad B, Rafiei M, Mondanizadeh M. [Bioinformatic prediction of miRNAs targeting Notch1 and HBx genes in chronic hepatitis B-induced hepatocellular carcinoma (Pertsian)]. Arak Med Univ J. 2017; 19(12):89-101. https://www.sid.ir/fa/journal/ViewPaper.aspx?!D=287922

[19] Rostamian Delavar M, Baghi M, Yadegari E, Ghaedi K. [Bioinformatic prediction and introducing of some targeting microRNAs of Sirt1 and Bcl2 genes in model of Parkinson's disease (Pertsian)]. J Arak Univ Med Sci. 2017; 20(8):61-72. https://www.researchgate.net/publication/324476337

[20] Li M, Ren CX, Zhang JM, Xin XY, Hua T, Wang HB, et al. The effects of miR-195-5p/MMP14 on proliferation and invasion of cervical carcinoma cells through TNF signaling pathway based on bioinformatics analysis of microarray profiling. Cell Physiol Biochem. 2018; 50(4):1398-413. [DOI:10.1159/000494602] [PMID]

[21] Zhou C, Shen L, Mao L, Wang B, Li Y, Yu H. MiR-92a is upregulated in cervical cancer and promotes cell proliferation and invasion by targeting FBXW7. Biochem Biophys Res Commun. 2015; 458(1):63-9. [DOI:10.1016/j.bbrc.2015.01.066] [PMID]

[22] Li N, Cui T, Guo W, Wang D, Mao L. MiR-155-5p accelerates the metastasis of cervical cancer cell via targeting TP53INP1. Onco Targets Ther. 2019; 12:3181-96. [DOI:10.2147/OTT.S193097] [PMID] [PMCID]

[23] Toroghi F, Toroghi Y. [Bioinformatics analysis to predict potential microRNAs inhibiting processes of angiogenesis in cancer (Pertsian)]. J Gorgan Univ Med Sci. 2017; 19(1):83-8. http://goums.ac.ir/journal/article1-3021-fa.html

[24] Li J, Yu L, Shen Z, Li Y, Chen B, Wei W, et al. MiR-34a and its novel target, NLRC5, are associated with HPV16 persistence. Infect Genet Evol. 2016; 44:293-9. [DOI:10.1016/j.meegid.2016.07.013] [PMID]

[25] Kim HJ, Cho H, Choi CH, Chung J, Hewitt SM, Hewitt SM. MicroRNA as biomarkers for cervical cancer. SM J Gynecol Obstet. 2015; 1(2):1-8. https://www.researchgate.net/publication/306538125

[26] Tian Q, Li Y, Wang F, Li Y, Xu J, Shen Y, et al. MicroRNA detection in cervical exfoliated cells as a triage for human papillomavirus-positive women. J Natl Cancer Inst. 2014; 106(9):dju241. [DOI:10.1093/jnci/ dju241] [PMID] [PMCID]

[27] Denny L. Cervical cancer: Prevention and treatment. Discov Med. 2012; 14(75):125-31. [PMID]

[28] Brown AJ, Trimble CL. New technologies for cervical cancer screening Best Pract Res Clin Obstet Gynaecol. 2012; 26(2):233-42. [DOI:10.1016/j. bpobgyn.2011.11.001] [PMID] [PMCID]

[29] Fitzmaurice C, Dicker D, Pain A, Hamavid H, Moradi-Lakeh M, Maclntyre MF, et al. The global burden of cancer 2013. JAMA Oncol. 2015 1(4):505-27. [DOI:10.1001/jamaoncol.2015.0735] [PMID] [PMCID]

[30] Lu J, Getz G, Miska EA, Alvarez-Saavedra E, Lamb J, Peck D, et al. MicroRNA expression profiles classify human cancers. Nature. 2005; 435(7043):834. [DOI:10.1038/nature03702] [PMID]

[31] McManus MT. MicroRNAs and cancer. Semin Cancer Biol. 2003 13(4):253-8. [DOI:10.1016/S1044-579X(03)00038-5]

[32] Le Quesne J, Caldas C. Micro-RNAs and breast cancer. Mol Oncol. 2010 4(3):230-41. [DOI:10.1016/j.molonc.2010.04.009] [PMID] [PMCID] 
[33] Ritchie W, Rasko JE, Flamant S. MicroRNA target prediction and validation. InMicroRNA Cancer Regulation. Berlin: Springer; 2013. pp. 39-53. [DOI:10.1007/978-94-007-5590-1_3] [PMID]

[34] Mo MH, Chen L, Fu Y, Wang W, Fu SW. Cell-free circulating miRNA biomarkers in cancer. J Cancer. 2012; 3:432-48. [DOI:10.7150/jca.4919] [PMID] [PMCID]

[35] Moradi N, Paryan M, Khansarinejad B, Sarmadian H, Mondanizadeh M. Plasma level of miR-5193 as a novel biomarker for diagnosis of HBVrelated hepatocellular carcinoma. Hepat Mon. 2019; 19(2):e84455. [DOI:10.5812/hepatmon.84455]

[36] Aghaee-Bakhtiari SH, Arefian E, Soleimani M, Mirab Samiee S, Noorbakhsh F, Mahdian R, et al. [Bioinformatic evaluations for locating the microRNA suppressing PI3K/AKT Pathway and analysis in prostate cancer cell lines (Persian)]. Pathobiol Res. 2015; 17(4):1-12. https://mjms. modares.ac.ir/article-30-11898-en.pdf

[37] Xia YF, Pei GH, Wang N, Che YC, Yu FS, Yin FF, et al. MiR-3156-3p is downregulated in HPV-positive cervical cancer and performs as a tumorsuppressive miRNA. Virol J. 2017; 14(1):20. [DOI:10.1186/s12985-0170695-7] [PMID] [PMCID]

[38] Honegger A, Schilling D, Bastian S, Sponagel J, Kuryshev V, Sültmann H, et al. Dependence of intracellular and exosomal microRNAs on viral E6/ E7 oncogene expression in HPV-positive tumor cells. PLoS Pathog. 2015; 11(3):e1004712. [DOI:10.1371/journal.ppat.1004712] [PMID] [PMCID]

[39] Wang X, Wang HK, Li Y, Hafner M, Banerjee NS, Tang S, et al. microRNAs are biomarkers of oncogenic human papillomavirus infections. Proc Natl Acad Sci. 2014; 111(11):4262-7. [DOI:10.1073/pnas.1401430111] [PMID] [PMCID]

[40] Song R, Cong L, Ni G, Chen M, Sun H, Sun Y, et al. MicroRNA-195 inhibits the behavior of cervical cancer tumors by directly targeting HDGF. Oncol Lett. 2017; 14(1):767-75. [DOI:10.3892/ol.2017.6210] [PMID] $[\mathrm{PMCID}]=$ 
This Page Intentionally Left Blank 